Wright State University

CORE Scholar

7-1-2006

\title{
Radiative Recombination and Ultralong Exciton Photoluminescence Lifetime in GaN Freestanding Film Via Two- Photon Excitation
}

Yongchun Zhong

Kam Sing Wong

Weili Zhang

David C. Look

Wright State University - Main Campus, david.look@wright.edu

Follow this and additional works at: https://corescholar.libraries.wright.edu/physics

Part of the Physics Commons

\section{Repository Citation}

Zhong, Y., Wong, K. S., Zhang, W., \& Look, D. C. (2006). Radiative Recombination and Ultralong Exciton Photoluminescence Lifetime in GaN Freestanding Film Via Two-Photon Excitation. Applied Physics Letters, 89 (2), 22108.

https://corescholar.libraries.wright.edu/physics/93

This Article is brought to you for free and open access by the Physics at CORE Scholar. It has been accepted for inclusion in Physics Faculty Publications by an authorized administrator of CORE Scholar. For more information, please contact library-corescholar@wright.edu. 


\title{
Radiative recombination and ultralong exciton photoluminescence lifetime in GaN freestanding film via two-photon excitation
}

\author{
Yongchun Zhong and Kam Sing Wong ${ }^{\text {a) }}$ \\ Department of Physics, Hong Kong University of Science and Technology, Kowloon, Hong Kong, \\ People's Republic of China \\ Weili Zhang \\ School of Electrical and Computer Engineering, Oklahoma State University, Oklahoma 74078 \\ D. C. Look \\ Semiconductor Research Center, Wright State University, Dayton, Ohio 45435 \\ and Materials and Manufacturing Directorate, Air Force Research Laboratory, Wright-Patterson AFB, \\ Ohio 45433
}

(Received 11 April 2006; accepted 2 June 2006; published online 10 July 2006)

\begin{abstract}
We have measured the photoluminescence (PL) lifetime of a freestanding GaN film using one-photon and two-photon excitations to demonstrate the dramatic difference in exciton recombination dynamics at the surface and in the bulk. An ultralong exciton PL lifetime of $17.2 \mathrm{~ns}$ at $295 \mathrm{~K}$ is observed from a GaN freestanding film using two-photon excitation, whereas less than 100 ps lifetime is observed for one-photon excitation, suggesting that nonradiative processes from surface defects account for the short PL lifetime measured. A monotonic increase in two-photon excited PL lifetime with increasing temperature and the linear dependence of the exciton lifetime with emission wavelength show good agreement with the theoretical predictions, indicating that radiative recombination dominates for bulk excited state relaxation processes. (C) 2006 American Institute of Physics. [DOI: 10.1063/1.2219399]
\end{abstract}

$\mathrm{GaN}$ is an emerging candidate material for fabrication of a variety of optical and electrical semiconductor devices having good high-frequency and high-temperature operational characteristics. ${ }^{1}$ GaN films of good optical quality grown by molecular beam epitaxy (MBE), metalorganic chemical vapor deposition (MOCVD), and hydride vapor phase epitaxy (HVPE) have been reported in recent years. ${ }^{2-5}$ The significant progress in growth techniques generates considerable interest in the photoluminescence (PL) excitonic lifetime as a probe to study sample quality. ${ }^{6-13}$

It is well known that surface defects can act as nonradiative recombination centers, and thus can affect the lifetimes of photogenerated carriers as well as the performances of certain electronic devices. ${ }^{14}$ All the time-resolved experiments performed so far show a band edge PL lifetime of a few tens to a few hundreds of picoseconds, indicating strong nonradiative recombination processes. ${ }^{6-13}$ The longest room temperature PL lifetime ever reported is about $2 \mathrm{~ns}$ for a GaN film grown on a TiN porus network template, but even in this case it has a large fast decay component with a decay time constant of about 400 ps. ${ }^{13}$ These PL experiments were done using above-band-gap excitation, and because of the very short absorption depth in this case the surface defects will strongly quench the excited carriers and excitons and consequently will produce a very short PL decay. One way to avoid this nonradiative process at the surface and study directly the intrinsic radiative recombination is by using twophoton excitation to generate carriers within the bulk in a thick GaN film. In this letter, we report on the optical characterization and time-resolved studies of a freestanding GaN film. A room temperature exciton PL decay time of $17.2 \mathrm{~ns}$

\footnotetext{
${ }^{\text {a) }}$ Author to whom correspondence should be addressed; electronic mail: phkswong@ust.hk
}

using two-photon excitation is the longest ever reported for a GaN film. Furthermore, an observed monotonic increase of the PL lifetime versus temperature indicates that radiative recombination dominates from 8 to $295 \mathrm{~K}$.

The experiments were carried out on a $250-\mu$ m-thick freestanding GaN film grown on a (0001) sapphire substrate by HVPE and then separated from the sapphire by laser liftoff. ${ }^{15}$ A femtosecond titanium-sapphire oscillator and a regenerative amplifier were used as the excitation sources.

Figure 1 shows the one- and two-photon excitation PL spectra of the sample at different temperatures. The dotted line shows the PL spectrum excited with the $355 \mathrm{~nm}$ laser (one-photon excitation). At a temperature of $8 \mathrm{~K}$, the PL spectrum is dominated by the bound-exciton emission peak at $3.4727 \mathrm{eV}$, denoted by $D^{0} X$. There is a weak free $A$ exciton emission at $3.4798 \mathrm{eV}$, denoted by $E_{X}{ }^{A}$. The spectrum also includes two weak luminescence lines attributable to a bound-exciton longitudinal-optical (LO) phonon replica at $3.3807 \mathrm{eV}$, and a free-exciton-LO phonon replica at $3.3882 \mathrm{eV}$, denoted by $E_{D}$ - $\mathrm{LO}$ and $E_{X}-\mathrm{LO}$, respectively. At higher temperatures, the excitonic and exciton-LO phonon replica lines broaden and are redshifted. Furthermore, free exciton emissions (i.e., $E_{X}{ }^{A}, E_{X}$-LO) are greatly enhanced and the bound exciton emissions (i.e., $D^{0} X, E_{D}$-LO) strongly suppressed due to ionization of the neutral donors at higher temperature. The observed temperature dependence of the $E_{D}$-LO and $E_{X}$-LO peaks are consistent with those reported for heteroepitaxial GaN films. ${ }^{16}$ The peak at $3.4476 \mathrm{eV}$ is in the region of two-electron $\left(D^{0} X\right)_{n=2}$ transitions; however, its intensity appears to be too large for a two-electron transition. The low energy peaks at 3.2594, 3.2898, and $3.2974 \mathrm{eV}$ are likely due to donor-acceptor-pair (DAP) emission and twophonon assisted exciton emissions (i.e., $E_{D}-2 \mathrm{LO}$ and $\left.E_{X}-2 \mathrm{LO}\right)$ respectively. ${ }^{16} \mathrm{~A}$ detailed spectroscopic assignment 


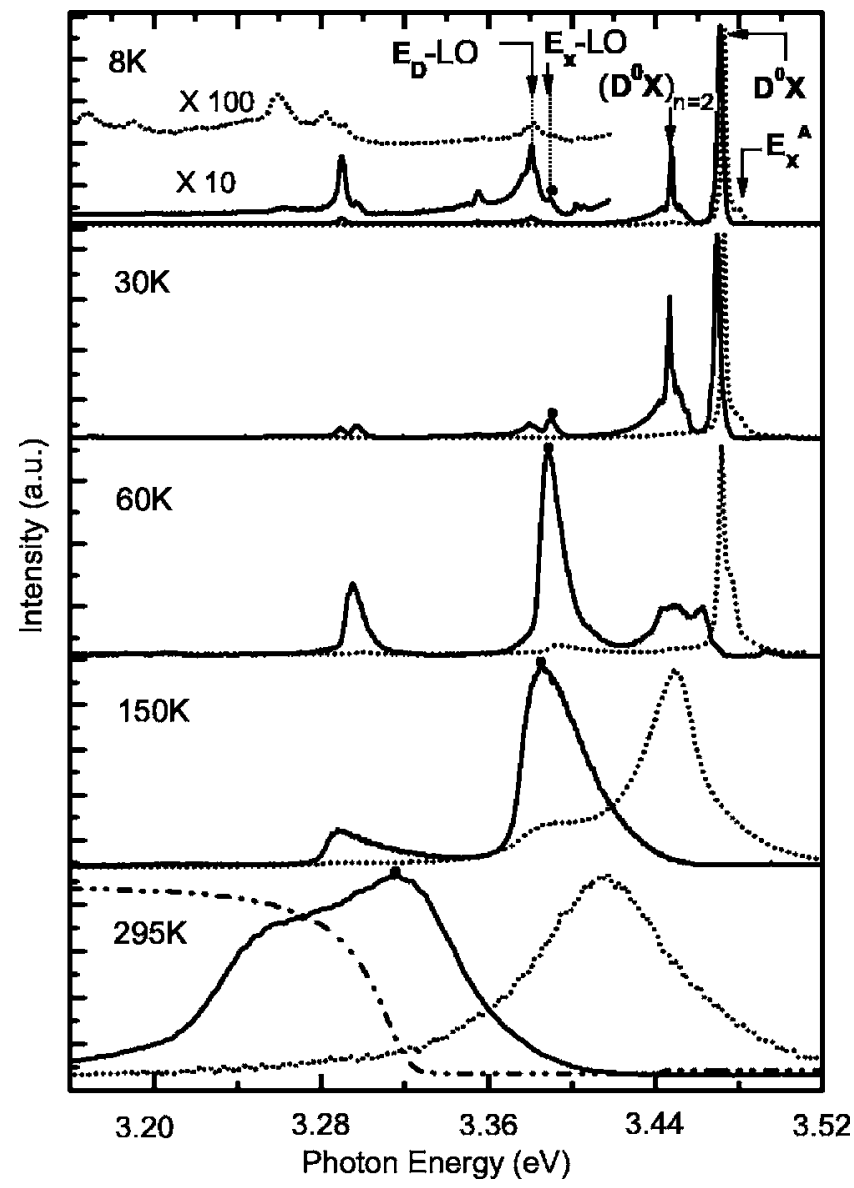

FIG. 1. The one- and two-photon-excited PL spectra at different temperatures. The dotted lines represent the one-photon excitation case and the solid lines, the two-photon-excitation case. The peaks marked with filled circles show the evolution of the exciton-LO-phonon emission peak. The dot-dash line is the transmission of the GaN film showing that the two-photon-excited PL peak is at the transmission edge of the sample.

of the PL peaks at low temperatures for strain-relaxed GaN films can be found in Ref. 17.

The solid lines show the PL spectrum excited with the $710 \mathrm{~nm}$ laser light for generating carriers/excitons within the bulk. The excitonic emission bands of the two-photoninduced PL are all redshifted compared to those resulting from one-photon excitation. This redshift is due to self-absorption. ${ }^{18}$

Figures 2(a) and 2(b) show the PL decay at various temperatures for one-photon-excited and two-photon-excited exciton emission peaks, respectively. A drastic difference in decay times is seen for the two cases. For the one-photonexcited PL, the emission lifetime decreases with increasing temperature and exhibits a multicomponent exponential decay, with decay time constants ranging from a few tens to a few hundreds of picoseconds. This behavior suggests rapid capture and nonradiative recombination resulting from surface defects. On the other hand, the two-photon-excited PL lifetime increases with increasing temperature, opposite to the one-photon case. It indicates that the two-photon exciton emission is dominated by radiative recombination. It is well known that self-absorption or photon recycling may influence the true PL lifetime. ${ }^{19-21}$ However, the low excitation intensities $\left(\Delta n<10^{16} \mathrm{~cm}^{-3}\right)$ used here ${ }^{20}$ and a single exponential decay with nanosecond decay time can rule out photon recycling as a contribution to PL lifetime. ${ }^{21}$
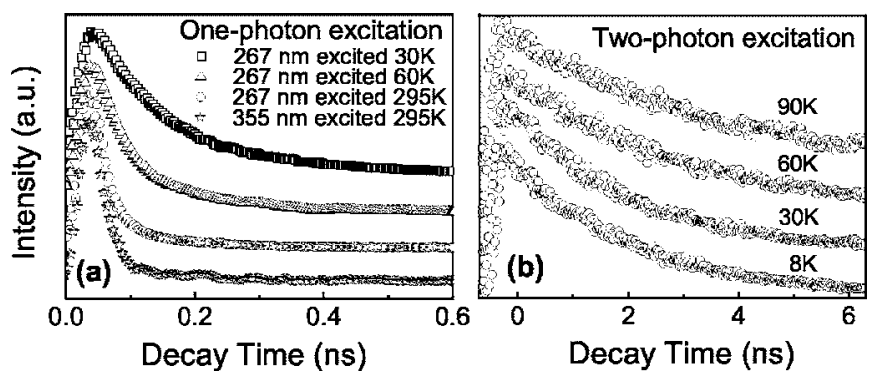

FIG. 2. (a) The PL decay of the one-photon-excited exciton emission peak at various temperatures. The room temperature PL decay times for both the 267 and $355 \mathrm{~nm}$ excitations are about the same. The $355 \mathrm{~nm}$ excitation intensity is more than three orders of magnitude lower than that using $267 \mathrm{~nm}$ excitation. This indicates that the fast PL decay is not a result of a nonlinear recombination process (such as Auger) due to the higher excitation intensity at $267 \mathrm{~nm}$. (b) The two-photon-excited exciton emission decay at various temperatures.

Figure 3 shows the two-photon-excited emission lifetime $\tau$ of the impurity-bound/free exciton and exciton-LO phonon replica as a function of temperature. A monotonic increase in $\tau$ with increasing temperature is seen. The inset (a) shows the emission decay at 8, 120, and $295 \mathrm{~K}$. A single exponential decay can be fitted to the time-resolved PL data for all temperatures. At room temperature $(295 \mathrm{~K})$, an ultralong exciton PL lifetime of 17.2 ns is observed.

Several models of radiative recombination processes for excitons have been developed by various authors. ${ }^{22-24}$ Assuming a Boltzmann distribution in kinetic energy space, the fraction $r$ of excitons with a kinetic energy smaller than $\Delta E$ is given $b^{23}$

$$
r(T)=\frac{2}{\sqrt{\pi}} \int_{0}^{\Delta E / k_{B} T} \sqrt{\varepsilon} e^{-\varepsilon} d \varepsilon .
$$

The temperature-dependent radiative lifetime is then

$$
\tau(T)=\tau_{0} / r(T)
$$

where $\tau_{0}$ is the radiative lifetime at temperature $0 \mathrm{~K}$. Equations (1) and (2) are fitted to the exciton-LO phonon

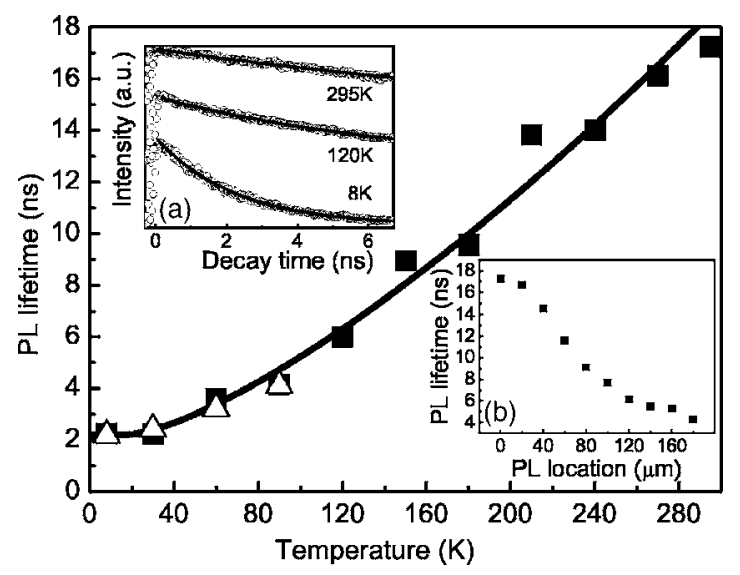

FIG. 3. The two-photon-excited exciton and exciton-LO-phonon emission lifetime as a function of temperature. The hollow triangles denote the experimental exciton emission, while the solid squares represent the experimental exciton-LO-phonon emission. As expected, the PL lifetimes of exciton and exciton-LO-phonon emission are about the same. The black curve is the theoretically fitted curve. Figure inset (a) shows the emission decay at different temperatures and the solid line is the single-exponential fit to the data and (b) shows the PL lifetime as a function of the location of the PL spot inside the sample. 


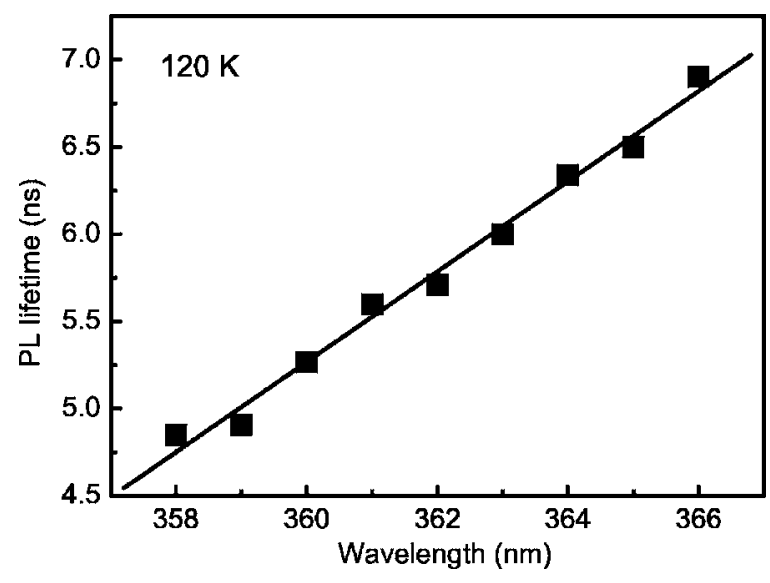

FIG. 4. The exciton lifetime as a function of emission wavelengths at $120 \mathrm{~K}$.

lifetime with $\tau_{0}$ and $\Delta E$ as fitting parameters. The solid curve in Fig. 3 shows good agreement with the experimental data for $\tau_{0}=2.18 \mathrm{~ns}$ and $\Delta E=8.4 \mathrm{meV}$. The parameter $\Delta E$ is related to the emission lifetime and it is about twice that of the low-temperature exciton linewidth $(5.3 \mathrm{meV}$, Fig. 1) deduced from Gaussian fitting. The theoretical curve shows good agreement with both the exciton and exciton-LOphonon emissions.

The radiative lifetime $\tau$ of the exciton state is given by ${ }^{23}$

$$
\tau=2 \pi \varepsilon_{\mathrm{o}} m_{\mathrm{o}} c^{3} / n e^{2} \omega^{2} f=\left(\pi \varepsilon_{\mathrm{o}} m_{\mathrm{o}} c^{2} \hbar V / n e^{2} E_{p} a_{x}^{3}\right) \lambda,
$$

where $f=E_{p} / \pi \hbar \omega\left(V / a_{x}^{3}\right)$ is the oscillator strength of the optical transition, $E_{p}$ is the interband momentum matrix element, $V$ is the volume of the unit cell, $a_{x}$ is the exciton radius, $n$ is the refractive index, and the other symbols have their usual meaning. Equation (3) shows that the radiative lifetime $\tau$ is proportional to the emission wavelength $\lambda$. Figure 4 shows the measured $\tau$ as a function of $\lambda$ and a clear linear dependence is observed as predicted by the theory.

The inset (b) of Fig. 3 shows the two-photon-excited PL lifetime as a function of the location of a focused laser excitation beam moved along the cross section of the GaN film. The location "0" is near the center of the sample and increasing distance means that the focused spot is moving toward the sample surface. Inset (b) shows the PL lifetime at the center location, which has the longest lifetime. The PL lifetime decreases as the spot moves away from the center of the sample and has the shortest lifetime ( $\sim 4.5 \mathrm{~ns})$ at the surface. One must note that the objective used for the two-photon excitation experiment had a numerical aperture of 0.1 and therefore the depth of field of the excitation spot was over $70 \mu \mathrm{m}$. Thus, there is a substantial excitation volume within the sample even when the focusing is apparently at the surface. Still, this result clearly shows that the influence of the surface defects becomes stronger for excitation nearer to the sample surface.

In conclusion, exciton recombination dynamics in a freestanding GaN film grown by HVPE have been studied at various temperatures using one- and two-photon excitations. The studies show that in the one-photon-excitation case, the exciton emission is dominated by nonradiative recombina- tion due to surface defects, whereas in the two-photonexcitation case, the nonradiative surface-recombination process is nearly absent and radiative recombination is the dominant mechanism from 8 to $295 \mathrm{~K}$. At room temperature, the PL lifetime is up to $17.2 \mathrm{~ns}$, which is the longest ever reported for GaN. The monotonic increase in PL lifetime with increasing temperature and the linear dependence of the exciton lifetime with emission wavelength are in excellent agreement with theoretical predictions for radiative recombination processes.

The authors would like to thank S. S. Park of Samsung for supply of the GaN sample. One of the authors (D.C.L.) was supported by AFOSR Grant No. F49620-03-1-0197 and Air Force Contract No. F33615-00-C-5402.

${ }^{1}$ S. Nakamura and G. Fasol, The Blue Laser Diode: GaN Based Light Emitters and Lasers (Springer, Berlin, 1997).

${ }^{2}$ S. Nakamura, T. Mukai, and M. Senoh, Appl. Phys. Lett. 64, 1687 (1994).

${ }^{3}$ W. Shan, T. J. Schmidt, X. H. Yanh, S. J. Hwang, J. J. Song, and B. Goldenberg, Appl. Phys. Lett. 66, 985 (1995).

${ }^{4}$ F. A. Ponce, D. P. Bour, W. Götz, N. M. Johnson, H. I. Helava, I. Grzegory, J. Jun, and S. Porowski, L. T. Romano, and N. M. Johnson, Appl. Phys. Lett. 68, 917 (1996).

${ }^{5}$ R. J. Molnar, W. Götz, L. T. Romano, and N. M. Johnson, J. Cryst. Growth 178, 147 (1997).

${ }^{6}$ W. Shan, X. C. Xie, J. J. Song, and B. Goldenberg, Appl. Phys. Lett. 67, 2512 (1995).

${ }^{7}$ J. S. Im, A. Moritz, F. Steuber, V. Härle, F. Scholz, and A. Hangleiter, Appl. Phys. Lett. 70, 631 (1997).

${ }^{8}$ S. Pau, Z. X. Liu, J. Kuhl, J. Ringling, H. T. Grahn, M. A. Khan, C. J. Sun, O. Ambacher, and M. Stutzmamn, Phys. Rev. B 57, 7066 (1998).

${ }^{9}$ M. Smith, G. D. Chen, J. Z. Li, J. Y. Lin, H. X. Jiang, A. Salvador, W. K. Kim, O. Aktas, A. Botchkarev, and H. Morkoç, Appl. Phys. Lett. 67, 3387 (1995).

${ }^{10}$ O. Brandt, J. Ringling, K. H. Ploog, H.-J. Wünsche, and F. Henneberger, Phys. Rev. B 58, R15977 (1998).

${ }^{11}$ Y. Narukawa, Y. Kawakami, S. Fujita, and S. Nakamura, Phys. Rev. B 59, 10283 (1999).

${ }^{12}$ G. E. Bunea, W. D. Herzog, M. S. Ünlü, B. B. Goldberg, and R. J. Molnar, Appl. Phys. Lett. 75, 838 (1999).

${ }^{13}$ Ü. Özgür, Y. Fu, Y. T. Moon, F. Yun, H. Morkoç, H. O. Everitt, S. S. Park, and K. Y. Lee, Appl. Phys. Lett. 86, 232106 (2005).

${ }^{14}$ A. Y. Polyakov, A. V. Govorkov, N. B. Smirnov, Z.-Q. Fang, D. C. Look, S. S. Park, and J. H. Han, J. Appl. Phys. 92, 5238 (2002); A. P. Zhang, L. B. Rowland, E. B. Kaminsky, V. Tilak, J. C. Grande, J. Teetsov, A. Vertiatchikh, and L. F. Eastman, J. Electron. Mater. 32, 388 (2003).

${ }^{15}$ S. S. Park, I.-W. Park, and S. H. Choh, Jpn. J. Appl. Phys., Part 2 39, L1141 (2000).

${ }^{16}$ S. J. Xu, W. Liu, and M. F. Li, Appl. Phys. Lett. 77, 3376 (2000); 81, 2959 (2002).

${ }^{17}$ B. J. Skromme, J. Jayapalan, R. P. Vaudo, and V. M. Phanse, Appl. Phys. Lett. 74, 2358 (1999).

${ }^{18}$ H. Wang, K. S. Wong, B. A. Foreman, Z. Y. Yang, and G. K. L. Wong, J. Appl. Phys. 83, 4773 (1998).

${ }^{19}$ P. Asbeck, J. Appl. Phys. 48, 820 (1977); R. J. Nelson and R. G. Sobers, ibid. 49, 6103 (1978).

${ }^{20}$ I. J. Blewett, N. R. Gallaher, A. K. Kar, and B. S. Wherett, J. Opt. Soc. Am. B 13, 779 (1996).

${ }^{21} \mathrm{Ph}$. Renaud, F. Raymond, B. Bensaïd, and C. Vèrié, J. Appl. Phys. 71, 1907 (1992).

${ }^{22}$ J. Feldmann, G. Peter, E. O. Göbel, P. Dawson, K. Moore, C. Foxon, and R. J. Elliott, Phys. Rev. Lett. 59, 2337 (1987).

${ }^{23}$ G. W. 't Hooft, W. A. J. A. van der Poel, L. W. Molenkamp, and C. T. Foxon, Phys. Rev. B 35, 8281 (1987).

${ }^{24}$ Lucio Claudio Andreani, A. d'Andrea, and R. del Sole, Phys. Lett. A 168, 451 (1992). 